\title{
Diversion, Misuse and Abuse of Prescription Drugs in Upper-Middle-Income Countries: Narrative Literature Review
}

\author{
${\text { Buyisile Chibi }{ }^{1,2} \& \text { Tivani P Mashamba-Thompson }}^{1}$ \\ ${ }^{1}$ Discipline of Public Health Medicine, School of Nursing and Public Health, University of KwaZulu-Natal, \\ Durban, South Africa \\ ${ }^{2}$ Human Sciences Research Council, Social Aspects of Public Health, Durban, South Africa \\ Correspondence: Buyisile Chibi, Discipline of Public Health Medicine, School of Nursing and Public Health, \\ University of KwaZulu-Natal, Durban 4001, South Africa; Human Sciences Research Council, Social Aspects of \\ Public Health, Durban 4001, South Africa. Tel: 27-31-242-5408. E-mail: chibuyi@gmail.com
}

Received: July 9, 2019 Accepted: August 5, 2019 Online Published: September 28, 2019

doi:10.5539/gjhs.v11n12p27 URL: https://doi.org/10.5539/gjhs.v11n12p27

\begin{abstract}
Globally, the diversion, misuse, and abuse of prescription drugs is a growing public health problem. This narrative review focused on factors influencing this problem in Upper-Middle-Income Countries. The literature reports that factors related to health systems and health providers such as retail pressure, insufficient consultation time, lack of screening tools, ineffective monitoring and surveillance systems, poor implementation of regulatory policies, lack of strict enforcement of prescribing policies as well as lack of specialized training perpetuate the problem. Evidence suggests that consumers with lack of appropriate knowledge about medication use, lack of awareness regarding potential risks, and poor attitude toward medication usage were more likely to engage into drug diversion and misuse. Based on a critical reflection of the literature, we propose a framework that outlines interventions needed to halt factors influencing drug diversion, misuse and abuse through a collaborative approach that will enable behavioural change and reduce the risk of harmful health outcomes.
\end{abstract}

Keywords: drug diversion, drug misuse, drug abuse, contributing factors

\section{List of abbreviations}

UMICs: Upper-middle-income countries

\section{Introduction}

Globally, medicines have made it possible to manage diseases for better health and well-being (Gumede-Moyo, Filteau, Munthali, Todd, \& Musonda, 2017; Singh, 2010). However, drug diversion, misuse and abuse is a growing public health problem (Cheah \& Chong, 2018). Here, we defined drug diversion as the illegal way of transferring controlled drugs from rightful owners to the black market for any use (Figure 1) (Inciardi, Surratt, Kurtz, \& Burke, 2006). Drug misuse is defined as the improper use of medicines with or without a health care provider's guidance but clearly not compliant to dosage instructions, time period or ways of drug administration therefore the risks of misuse are more significant than the benefits (Casati, Sedefov, \& Pfeiffer-Gerschel, 2012). Drug abuse is defined as the non-medical use of drugs in harmful amounts (Akerele \& Olupona, 2017). The United Nations Office of Drugs and Crime reports analgesics, tranquilizers, stimulants and antibiotics as the most commonly misused drugs (Cheah \& Chong, 2018). The World Drug Report documented about 190000 drug-related deaths in 2015 due to non-medical use (Merz, 2018). Meanwhile in 2017, the World Health Organization globally estimated an annual mortality rate of 10 million people by the year 2050 due to antibiotic resistance resulting from inappropriate antibiotic use (Pires, de Kraker, Tartari, Abbas, \& Pittet, 2017).

The main challenges with the prevention and reduction strategies wee: (1) there was little understanding of factors influencing medication diversion, misuse and abuse compared with illicit drugs; (2) due to health benefits of using pharmaceuticals their perceived risk was often not taken seriously and (3) policies, law enforcement, reduction and prevention strategies wee more focused, evaluated and known for substance abuse of which includes alcohol and illegal drugs (Carter-Francique, Hart, \& Cheeks, 2015). In this review, we provide a narrative account of factors influencing drug diversion, misuse abuse in relation to the health systems delivery, health care providers, pharmacists, and consumers in Upper-Middle-Income Countries (UMICs). Understanding the associated factors could better inform policy makers, health care providers and other relevant personnel for tailored made interventions for prevention and reduction of prescription drug diversion, misuse and abuse. 


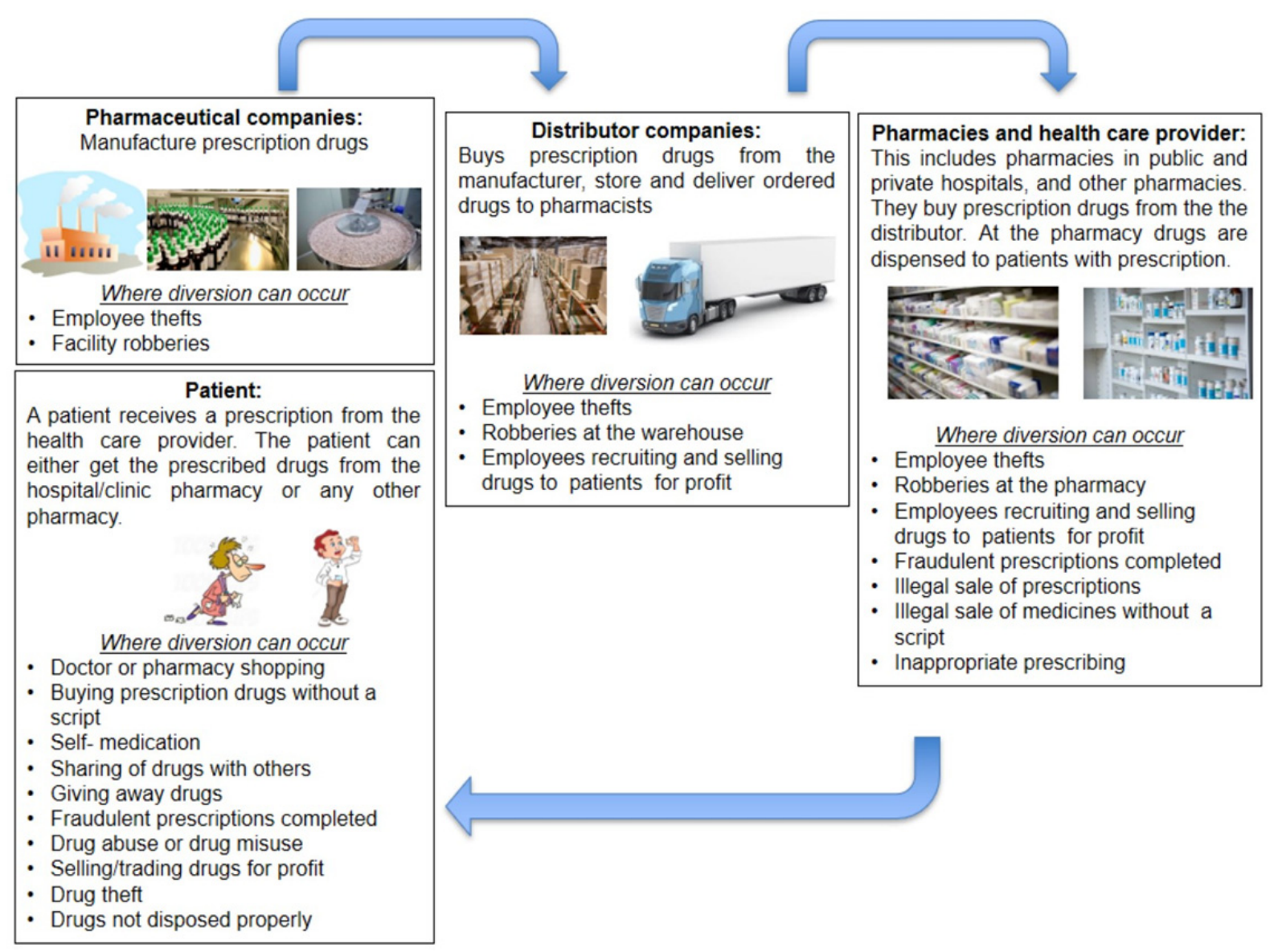

Figure 1. Illustration of drug diversion definition adapted from http://slideplayer.com/slide/237015/ (Jaber, Bulatova, Suyagh, Yousef, \& Wazaify, 2015)

\section{Search Strategy and Selection Criteria}

A search of recent (2014-2019) existing literature presenting factors contributing to drug diversion, misuse and abuse in UMICs was conducted from PubMed and EBSCO host databases. The main search terms were: medic* OR pharmac* OR prescri* OR drug* AND misuse OR diversion OR abuse AND factor*. The search was only limited to primary studies published in English reporting on factors influencing drug diversion, misuse and abuse in UMICs.

\section{Factors influencing Drug Diversion, Misuse and Abuse}

The factors influencing drug diversion, misuse and abuse include the following: (1) Health systems delivery, factors associated with (2) health care providers, (3) pharmacists and (4) consumers and patients.

\subsection{Health Systems Delivery}

Evidence shows that the health systems delivery factors such as the supply and demand for medicines and enforcement of regulations influences drug diversion, misuse and abuse in UMICs. Numerous medicines which carry health potential risks when misused wee readily available (T. Carney et al., 2018; Foley et al., 2018; Martinez et al., 2019) and easily accessible (Peng, Wang, Xu, Sun, \& Zhou, 2018; Shirzadeh, Shomoossi, \& Abdolahzadeh, 2016; X. Wang et al., 2017; Yaylaci et al., 2017) in most resource-limited settings. Results from two cross-sectional studies aimed at obtaining and analysing opinions and experiences of pharmacy staff/medical professionals regarding codeine misuse in South Africa revealed codeine misuse as a public health concern ( $\mathrm{T}$. Carney et al., 2018; Foley et al., 2018). These studies showed that non-prescription formulations with codeine were readily available in pharmacies and on the internet (T. Carney et al., 2018; Foley et al., 2018). However, up scheduling of codeine containing medicines to prescription only was opposed by most consumers in South Africans (Wells et al., 2018). Less than a quarter of medical professionals in South Africa confirmed having appropriate screening tools to assist with identifying codeine addictive behaviours among patients (Foley et al., 2018). 


\subsubsection{Supply of Medicines}

Evidence has shown that drug reps increase drug sales by consistently prodding prescribers to prescribe certain medicines to patients therefore translating into overprescribing (Peng et al., 2018). These have predisposed most pharmacies to sell medications to patients even when they do not have a valid doctors' prescription (Peng et al., 2018; Shirzadeh et al., 2016; X. Wang et al., 2017). In other countries, people were able to readily access prescription medications without a doctor's prescription. For example, in Turkey (Yaylaci et al., 2017) among patients receiving levothyroxine treatment and also in Iran (Shirzadeh et al., 2016) patients purchased tetracaine eye drops from pharmacies without a doctors' prescription. Most drug misusers reported pharmacies as their main source of pharmaceutical drugs and it was also emphasized that accessibility via online drug sale was ideal for some people since there were no tedious questions or documentation needed to facilitate a purchase (Van Hout, Rich, Dada, \& Bergin, 2017). Pharmacists in South Africa indicated a number of factors such as retail pressure, competitive commercial chains, insufficient time to interact and consult with clients, poor implementation of client screening tools, ineffective information provision models (monitoring and surveillance systems), insufficient resources and lack of consultation platforms influences their decision to supply over-the-counter codeine products to customers (Tara Carney et al., 2016). In South Africa, systems such as screening tools, interventions and referral structures were acknowledged however implementation of those systems were viewed as complex (Tara Carney et al., 2016). Challenges in the implementation of interventions and referrals were mainly the lack of awareness of referral structures and appropriate treatment centres (Tara Carney et al., 2016).

\subsubsection{Consumer Demand for Medications}

It is evident that there were numerous factors that influence consumers to misuse drugs. High prevalence of prescription non-compliance among consumers has been documented (de Oliveira et al., 2018; Peng et al., 2018; $\mathrm{X}$. Wang et al., 2017). For example, Chinese students would request antibiotic prescriptions during consultation when the health care provider has not initially offered them (Peng et al., 2018; X. Wang et al., 2017). Thus patients persistently pressurized prescribers to issue a prescription for antibiotics (Peng et al., 2018; X. Wang et al., 2017). Self-medicating behaviour was observed through home keeping of left-over antibiotics for future use (Peng et al., 2018; X. Wang et al., 2017; X. M. Wang, Zhou, \& Hesketh, 2016). Self-medicating was mostly influenced by inadequate knowledge about the potential risks of medicines (Peng et al., 2018; X. Wang et al., 2017; X. M. Wang et al., 2016). A study conducted in South Africa showed that drug misusers can exert manipulative behaviour which can influence healthcare workers to prescribe codeine containing products (Van Hout, Rich, et al., 2017). Drug misusers showed behaviours such as manipulating conversations and exaggerating symptoms with the aim of obtaining a prescription (Van Hout, Rich, et al., 2017).

\subsubsection{Regulations and Enforcement of Drug Sales}

Evidence suggests that there wass an increased access to prescription medications through over-the-counter purchases (Foley et al., 2018), lack of screening tools (Foley et al., 2018), lack of monitoring systems (Van Hout, Rich, et al., 2017), poor supervision (Foley et al., 2018), weak enforcement of regulations and policies (Foley et al., 2018) as drivers for drug diversion, misuse, and abuse in resource-limited settings. For example, Chinese students from a less developed province (Guizhou) were able obtain antibiotics from pharmacies without a script (Peng et al., 2018). About $45 \cdot 0 \%$ of medical professionals were unable to identify patients with medication misuse behaviours unless if the patient disclosed drug dependency (Foley et al., 2018). Prescribers reported issues related to over-the-counter sale of codeine products in drug stores and urged for improved systems to monitor drug sales at various drug stores (Foley et al., 2018). The availability of over-the-counter codeine containing products in drugs stores and also online poses a public health concern (Foley et al., 2018). About $85.3 \%$ of health care providers felt that this kind of medicines should be up-scheduled to prescription only (Foley et al., 2018). Interestingly, only $60.0 \%$ of prescribers routinely reviewed patients with codeine prescriptions (Foley et al., 2018). Drug misusers in South Africa reported that they were able to purchase these misused drugs at different outlets in different areas at various times to avoid been caught or identified (Van Hout, Rich, et al., 2017). Among the interviewed drug treatment providers in South Africa, only a few were aware of the 'Best-practice guidelines for the management of opioids use disorder' that was developed by South African Addiction Medicine Society (Parry, Rich, Van Hout, \& Deluca, 2017). In Malaysia, lack of institutional antibiotic guidelines was a driving factor to the continuous inappropriate antibiotic use in the hospital surgical wards (Mun Kit et al., 2015).

In summary, although the health systems were governed by regulations and policies two studies report poor implementation of regulatory policies(Tara Carney et al., 2016) and lack of strict enforcement of prescribing policies (Foley et al., 2018) as drivers. Moreover in terms of resources needed to assist with continuous monitoring, three studies report ineffective monitoring systems and ineffective surveillance systems (Tara Carney et al., 2016; Dada, Harker Burnhams, Van Hout, \& Parry, 2015; Foley et al., 2018). Despite the importance of adequate resources essential to identify potential medication misusers, three studies report lack of screening 
tools(Tara Carney et al., 2016; Van Hout, Rich, et al., 2017), poor supervision (Foley et al., 2018) and insufficient time to interact and consult with clients (Tara Carney et al., 2016) in the facilities. On the contrary, only one study reported retail pressure and competitive commercial chains as influencing factors (Tara Carney et al., 2016). On the other hand, lack of awareness of referral structures (Tara Carney et al., 2016) and lack of appropriate treatment centres (Tara Carney et al., 2016; Dada et al., 2015) were some of the barriers halting the epidemic. Lastly, factors related to the demand of medicines were mainly non-compliance to medication and to policies (Peng et al., 2018; Van Hout, Rich, et al., 2017; X. Wang et al., 2017; X. M. Wang et al., 2016).

\subsection{Factors Associated With Health Care Providers}

Disparities related to health care providers such as lack of specialized trainings, non-compliance to policies and economic incentives continuously affected the accessibility of medicines including drugs with high risk potential. For example, in China, overprescribing of antibiotics by health care providers was more common in rural areas and mainly facilitated by economic distress and misaligned incentives (Peng et al., 2018). A study in South Africa, reports that medical professionals prescribing codeine containing products were able to identify at least three patients with codeine dependence behaviour however only $43.0 \%$ of these practitioners were able to refer one patient to addiction services in the past month (Foley et al., 2018). In South Africa, addiction treatment providers highlighted that in a year about 41 patients were admitted to receive treatment however there were only few drug treatment centres in rural areas (Parry et al., 2017). Out of 20 treatment providers only two treatment providers had received specialist training where one was trained in Australia and the other through a conference (Parry et al., 2017). Similarly, most pharmacy staff reported that they had not undertaken any specialist training in substance use problems and indicated the need for public teaching and further trainings (T. Carney et al., 2018). A study in Jordan highlighted lack of suitable and effective communication between a health care provider and a parent (Farha, Suyagh, Alsakran, Alsous, \& Alefishat, 2016). A study in Malaysia mentioned that inappropriate antibiotic use was primarily governed by the lack of institutional antibiotic guidelines which negatively impacted administration time, treatment duration and choice of antibiotics to use (Mun Kit et al., 2015).

In summary, it was reported that health care providers wee faced with a lack of specialized training (T. Carney et al., 2018; Parry et al., 2017), non-compliance to policies (Peng et al., 2018), perverse economic incentives (Peng et al., 2018), and lack of institutional guidelines (Mun Kit et al., 2015), which continuously fuelled the problem. Although one study reported referring patients to the addiction services (Foley et al., 2018) and another admitting patients to receive treatment (Parry et al., 2017), both studies reported numbers below average. Lastly only one study documented lack of suitable and effective communication between a health care provider and a parent (Farha et al., 2016).

\subsection{Factors Associated With Pharmacists}

Medicinal drug sale with or without a prescription were prevalent in most countries. Pharmacy staffs (80.0 \%) wished to be involved in centralized monitoring systems for prescribers and dispensers (T. Carney et al., 2018; Jaber et al., 2015). Most pharmacy staff reported that they had not undertaken any specialist training in substance use problems and indicated the need for public teaching and further trainings (T. Carney et al., 2018). Contrary to that in Jordan, $63.6 \%$ of pharmacy students reported to have received training about drug misuse and abuse as part of pharmacy curriculum (Jaber et al., 2015). In Jordan about $35.4 \%$ of pharmacists acknowledged that drug sale of prescription drugs as an essential source of income in a drugstore (Jaber et al., 2015). Findings from a multi country study (including South Africa) among community pharmacists were that pharmacists felt that the pharmacy profession has been disrespected by clients (Tara Carney et al., 2016). Corruption among pharmacists especially the ones in the cities was reported in South Africa (Van Hout, Rich, et al., 2017).

In summary, it is reported that pharmacists were faced with lack of centralized monitoring systems for prescribers and dispensers(T. Carney et al., 2018; Jaber et al., 2015)and lack of specialist trainings(T. Carney et al., 2018). Lastly, pharmacists reported additional factors such as corruption (Van Hout, Rich, et al., 2017) and pressure to generate revenue(Jaber et al., 2015) as driving diversion.

\subsection{Factors Associated With Consumers and Patients}

Research show that there were socio-demographics factors such age(Farha et al., 2016; Haddad \& Ebada, 2017; Li et al., 2016; Yong-Kang, 2018), employment status/monthly income(Haddad \& Ebada, 2017; Yong-Kang, 2018), level of education(Farha et al., 2016; Haddad \& Ebada, 2017; Yong-Kang, 2018), marital status(Yong-Kang, 2018), area of residence(Yong-Kang, 2018) and well-being(Yong-Kang, 2018)driving drug diversion, misuse and abuse. For example, a study in China reported high levels of education as risk factor of inappropriate use of antibiotics (Peng et al., 2018). In Malaysia, lack of medicinal label viewing by consumers was found to contribute to the misuse of medicine.(Cheah \& Chong, 2018) On the other hand, age has an adverse effect on the ability of reading and knowing the medicinal label since every additional year in age was reported to decrease the ability of 
being aware of the medicine label by $0.4 \%$ (Yong-Kang, 2018). Meanwhile a study in South Africa reported that $29.0 \%$ of customers did not read the medicine pamphlet (Wells et al., 2018). A study in China reported that individuals with secondary education or tertiary education level were more likely to know the medication leaflet information than primary educated people (Yong-Kang, 2018). Although there was no significant relationship between the ability to read medicinal labels with gender, females were more likely to read than males (Wells et al., 2018). Contrary to the South African study, in China, males were more likely to know the medicine information pamphlet than females (Yong-Kang, 2018). In Jordan, patients with low level of education (no university degree) were less likely to ask for guidance from pharmacists, had greater chance of misusing drugs and were not ready to purchase expensive medicines (Haddad \& Ebada, 2017). In South Africa, 26.0\% of consumers reported that health care providers, family and friends were their main source of information (Wells et al., 2018).

\subsubsection{Behavioural Factors}

Different forms of childhood maltreatment or abuse have a significant negative impact to the psychosocial and behavioural aspect in the adulthood stage. Individuals experiencing codeine misuse and dependence in South Africa reported early life experiences (especially childhood maltreatment) as the contributing factor to their misuse behaviour (Van Hout, Norman, Rich, \& Bergin, 2017). Similarly, childhood maltreatment was prevalent among Chinese students who misused any prescription drugs (Guo et al., 2017; Lei et al., 2018). A study in China reported greater association between physical abuse and non-medical of drugs such as opioids, sedatives and any prescription drugs (Guo et al., 2017). Meanwhile emotional abuse, sexual abuse and emotional neglect were linked with greater risk of misusing opioids (Guo et al., 2017; Lei et al., 2018) and sedatives (Guo et al., 2017). Individuals who have experienced childhood physical neglect were more likely to engage in non-medical use of sedatives and any prescription medications (Guo et al., 2017). On the other hand, mental health problems such as depressive symptoms were significantly linked with drug misuse but there was no interaction effect of childhood maltreatment and depressive symptoms on non-medical use of prescription drugs (Guo et al., 2017).

Other factors that influenced most drug misusers/abusers were lack of awareness of their dependence since they were unacquainted with the potential abuse, harms and dependence as a result of inappropriate use of codeine containing formulas (Van Hout, Norman, et al., 2017). Students who stored antibiotics at home had 5 fold increased odds of self-medicating with antibiotics (X. Wang et al., 2017). A study in South Africa showed that during consultation, drug misusers were strong at manipulating conversations and exaggerating symptoms when requesting for a prescription of codeine products (Van Hout, Rich, et al., 2017). Most misusers had a history of illegal drug use, past experience with misusing medicinal drugs, past experience of different kinds of psychological problems, viewed the use of over-the-counter medicines as harmless and lastly most did not realize their addiction problem (Van Hout, Rich, et al., 2017).

\subsubsection{Knowledge, Attitude and Awareness}

Several studies showed low level of education, lack of knowledge and lack of awareness as contributing factors for inappropriate medication usage (Gu et al., 2015; Jaber et al., 2015; Wati Ngadimon, Islahudin, Hatah, Mohamed Shah, \& Makmor-Bakry, 2015; Yong-Kang, 2018). For instance, Chinese students with lower knowledge scores were more likely to self-medicate with antibiotics, to consult a medical professional when sick with self-limiting illnesses, to be prescribed antibiotics and to request the health care provider to prescribe antibiotics if the health care provider initially was unwilling when compared with students with higher knowledge (X. Wang et al., 2017). Another study in China reported poor knowledge related to antibiotics and their use (Yaylaci et al., 2017). More than 50.0 \% of codeine medicine users in South Africa viewed codeine containing mixtures as safe medicines (Wells et al., 2018). Meanwhile another study in South Africa reported that $89.0 \%$ of medical professional believed patients thought over-the-counter codeine containing mixtures were safe for use (Foley et al., 2018). In support to this, $95 \cdot 0 \%$ of medical professionals in South Africa perceived that patients did not realize the potential risks of drug dependency related to misuse of over-the-counter codeine containing products (Foley et al., 2018). A study in Jordan reported that fathers were more aware of the appropriate use of antibiotics when compared to mothers (Farha et al., 2016). Pharmacists from South Africa reported that most of their clients do not know the product names but they could identify and describe the requested medication based on their colour, appearance and packaging (Tara Carney et al., 2016). A study in Malaysia among secondary students reported low level of knowledge (71.13\%) about antibiotic usage however high knowledge scores were associated with a more negative experience with antibiotic usage (Wati Ngadimon et al., 2015).

Gu et al. (2015) study aimed to identify and compare factors affecting the knowledge of, attitude towards and use of antibiotics between urban and rural residents in China reported that greater knowledge was related with female, increasing age, higher level of education, poor health, a family member engaged in medical related work (Gu et al., 2015). However better attitude towards antibiotics was significantly associated with urban residency, higher level of education, a high income, better health, a family member engaged in medical related work (Gu et al., 2015). 
Individuals with higher level of education than primary school were more likely to be aware of the medicine label (Yong-Kang, 2018).

\subsubsection{Motivational Contexts}

Although, codeine containing mixtures wee meant to alleviate pain, $6.0 \%$ of costumers in South Africa reported using these medicines for relaxing purposes (Wells et al., 2018). Medical professionals believed that patients thought they can use over-the-counter codeine products recreationally (Foley et al., 2018). Motives of using codeine based drugs were initially based on bio-psycho-social motives, for euphoric effects, for relaxation effect and used as a coping mechanism however their continued used led to addiction and dependency (Van Hout, Rich, et al., 2017). Parents gave their children non-prescribed antibiotics since the child previously had similar symptoms or it was recommended by a pharmacist (Farha et al., 2016).

In summary, factors related to consumers were significant since consumers controlled the rate of demand of medicines. This review show that consumers with low level of education (Farha et al., 2016; Haddad \& Ebada, 2017; Yong-Kang, 2018), lack of appropriate knowledge about medication use (Tara Carney et al., 2016; Jaber et al., 2015; Peng et al., 2018; X. Wang et al., 2017; Yaylaci et al., 2017), lack of awareness regarding potential risks (Tara Carney et al., 2016; Farha et al., 2016; Jaber et al., 2015; Yong-Kang, 2018) and poor attitude toward medication usage(Gu et al., 2015; Jaber et al., 2015) were more likely to engage into drug diversion and misuse. Nevertheless consumers had additional factors such as early life experiences (Van Hout, Norman, et al., 2017), mental health problems (Guo et al., 2017),recreational use purpose (Foley et al., 2018; Van Hout, Rich, et al., 2017; Wells et al., 2018) and biopsychosocial purposes (Van Hout, Rich, et al., 2017) hence facilitating continuous drug diversion and misuse.

\section{Discussion}

This narrative review presented an overview of literature on contributing factors for drug diversion, misuse and abuse in UMICs. Health systems delivery in all countries were lacking due to numerous factors such as poor implementation of regulatory policies (Tara Carney et al., 2016), lack of strict enforcement of prescribing policies (Foley et al., 2018), ineffective monitoring and surveillance systems (Tara Carney et al., 2016; Dada et al., 2015; Foley et al., 2018), lack of screening tools (Tara Carney et al., 2016; Van Hout, Rich, et al., 2017), poor supervision(Foley et al., 2018), insufficient time to interact and consult with clients(Tara Carney et al., 2016), retail pressure and competitive commercial chains(Tara Carney et al., 2016), lack of awareness of referral structures(Tara Carney et al., 2016), lack of appropriate treatment centres(Tara Carney et al., 2016; Dada et al., 2015) and lastly non-compliant to policies (Peng et al., 2018; Van Hout, Rich, et al., 2017; X. Wang et al., 2017 ; X. M. Wang et al., 2016) therefore hindering the achievement to improved health outcomes. Generally, most health care providers and pharmacists lacked specialized trainings (T. Carney et al., 2018; Parry et al., 2017) and were non-compliant to policies (Peng et al., 2018; Van Hout, Rich, et al., 2017). Besides, other facilities lacked institutional guidelines for appropriate prescribing rationale (Mun Kit et al., 2015). Patient referral to drug treatment centres was a challenge (Foley et al., 2018; Parry et al., 2017). On the other hand, consumers with low level of education (Farha et al., 2016; Haddad \& Ebada, 2017; Yong-Kang, 2018), lack of appropriate knowledge about medication use (Abu Taha et al., 2016; Tara Carney et al., 2016; Jaber et al., 2015; Peng et al., 2018 ; X. Wang et al., 2017; Yaylaci et al., 2017), lack of awareness regarding potential risks (Tara Carney et al., 2016; Farha et al., 2016; Jaber et al., 2015; Yong-Kang, 2018), poor attitude toward medication usage (Abu Taha et al., 2016; Gu et al., 2015; Jaber et al., 2015) were disproportionally burdened by diversion and misuse. Meanwhile consumers with early life experiences (Van Hout, Norman, et al., 2017), mental health problems (Guo et al., 2017), recreational use purpose (Foley et al., 2018; Van Hout, Rich, et al., 2017; Wells et al., 2018) and biopsychosocial purposes of use (Van Hout, Rich, et al., 2017) were more affected.

\subsection{Critical Reflections and Synthesis}

In this section, a proposed framework based on a critical reflection of the extant literature is presented. It is evident that drug diversion, misuse and abuse needs to be addressed and dealt with collectively. The health systems, consumers, prescribers and dispensers should collectively work together with the purpose of improving the health outcomes. Interventions to halt the widespread of abuse and avoid the consequences related to inappropriate use of medication are urgently needed, particularly in diseases-burdened countries. These could help reduce the increasing drug resistant strains for tuberculosis, human immunodeficiency virus, and some bacterial infections. Based on the above evidence, we propose a framework to enable the implementation of interventions to improve health outcomes (Figure 2). There is clearly a need for the development of appropriate screening tools to assist with identifying addictive behaviours among patients. There is a need for advanced monitoring tools, surveillance and integrated prescriber-dispenser data systems to monitor the rate of medication distribution to consumers. Incorporating tailored trainings for health providers to alert them of all medicines with potential risks of misuse 
and abuse could also be useful. In addition, strategies and interventions to address drug diversion, misuse and abuse among consumers are imperative to improve consumer knowledge, awareness and attitude toward the use of medications. It will be essential for policy makers to consider the association of factors between health systems, health provider, dispenser and consumer in order to fully address issues around this threat. Education campaigns and training platforms for health providers, pharmacists and consumers regarding the risks of diversion, misuse and abuse of medicines are warranted. Stakeholders such as researchers, health providers and pharmacy service providers need to work together to ensure appropriate monitoring and evaluation of the interventions in line with relevant policies and guidelines. The proposed interventions may help bring awareness, improve consumer knowledge and attitude toward medicines use and potential risks is warranted to be a joint intervention between the health care providers, the pharmacists, and the public.

\section{Conclusion}

The reviewed literature shows that diversion and misuse of over-the-counter and prescription drugs is a growing problem in resource-limited settings. However, it also suggests that considerable attention should be paid to the health systems, consumers, prescribers, and dispensers to strengthen the implementation and enforcement of regulations, policies, guidelines, monitoring systems, monitoring tools and the emphasis of appropriate prescribing, and dispensing of medicines. Better public education aimed at improving consumer knowledge, awareness, and attitude of appropriate medication use is one approached that may encourage the prudent use medicinal drugs. In addition, monitoring and evaluation of health systems delivery, health care providers, pharmacists and consumers/patients is needed to explore the extent, and risk factors associated with drug misuse in resource-limited settings, particularly those with high disease burden.

\section{Declarations}

\subsection{Ethics Approval and Consent to Participate}

Not applicable

\subsection{Consent for Publication}

Not applicable

\subsection{Data Availability}

Not applicable

6.4 Funding

University of KwaZulu-Natal for financial support, recourses and materials. National Research Foundation (NRF) for financial support.

\subsection{Authors' Contributions}

$\mathrm{BC}$ conceptualized and designed the study. $\mathrm{BC}$ conducted the literature search. BC prepared the first manuscript draft including figures which was reviewed by TPM-T several times until ready for submission.

\subsection{Acknowledgements}

The authors would like to thank the Human Sciences Research Council, especially the HSRC Information Consultant Team for their assistance with accessing full-text.

\section{Competing Interests Statement}

The authors declare that there are no competing or potential conflicts of interest. 


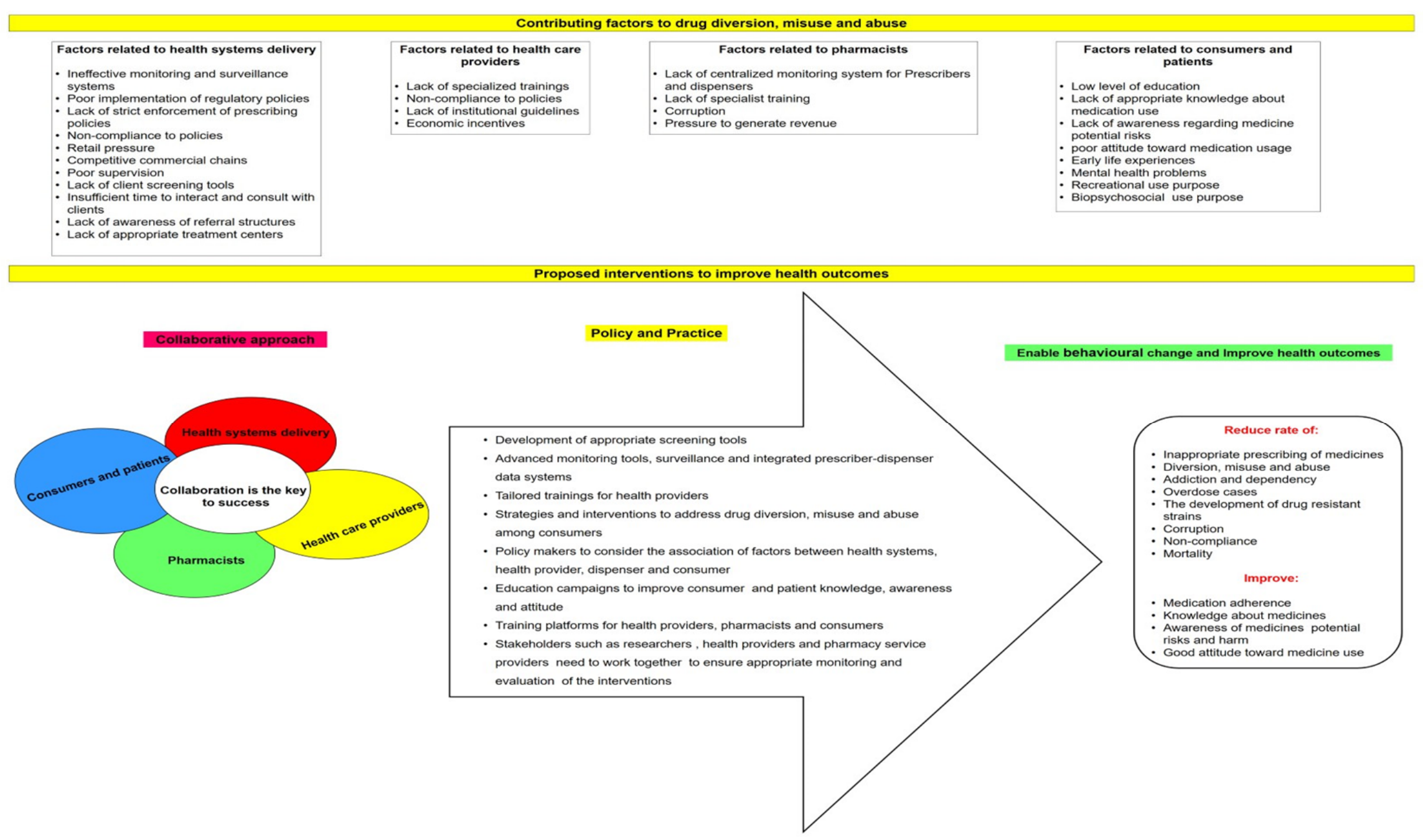

Figure 2. The proposed framework outlines interventions needed to halt drug diversion, misuse and abuse through a collaborative approach to influence policy and practice in-order to enable behavioural change and improve health outcomes. 


\section{References}

Abu Taha, A., Abu-Zaydeh, A. H., Ardah, R. A., Al-Jabi, S. W., Sweileh, W. M., Awang, R., \& Zyoud, S. H. (2016). Public Knowledge and Attitudes Regarding the Use of Antibiotics and Resistance: Findings from a Cross-Sectional Study Among Palestinian Adults. Zoonoses \& Public Health, 63(6), 449-457. https://doi.org/10.1111/zph.12249

Akerele, E., \& Olupona, T. (2017). Drugs of Abuse. The Psychiatric clinics of North America, 40(3), 501-517. https://doi.org/10.1016/j.psc.2017.05.006

Carney, T., Wells, J., Bergin, M., Dada, S., Foley, M., McGuiness, P., . . Hout, M. (2016). A Comparative Exploration of Community Pharmacists' Views on the Nature and Management of Over-the-Counter (OTC) and Prescription Codeine Misuse in Three Regulatory Regimes: Ireland, South Africa and the United Kingdom. International Journal of Mental Health \& Addiction, 14(4), 351-369. https://doi.org/10.1007/s11469-016-9640-z

Carney, T., Wells, J., Parry, C. D. H., McGuinness, P., Harris, R., \& Van Hout, M. C. (2018). A comparative analysis of pharmacists' perspectives on codeine use and misuse - a three country survey. Substance abuse treatment, prevention, and policy, 13(1), 12. https://doi.org/10.1186/s13011-018-0149-2

Carter-Francique, A. R., Hart, A., \& Cheeks, G. (2015). Examining the value of social capital and social support for Black student-athletes' academic success. Journal of African American Studies, 19(2), 157-177. https://doi.org/10.1007/s12111-015-9295-z

Casati, A., Sedefov, R., \& Pfeiffer-Gerschel, T. (2012). Misuse of medicines in the European Union: a systematic review of the literature. European addiction research, 18(5), 228-245. https://doi.org/10.1159/000337028

Cheah, Y. K., \& Chong, Y. W. (2018). Factors influencing medication label viewing in Malaysia. Global health promotion, 25(1), 63-72. https://doi.org/10.1177/1757975916649135

Dada, S., Harker Burnhams, N., Van Hout, M. C., \& Parry, C. D. (2015). Codeine misuse and dependence in South Africa--learning from substance abuse treatment admissions. South African medical journal, 105(9), 776-779. https://doi.org/10.7196/SAMJnew.8172

de Oliveira, S. M., Altmayer, S., Zanon, M., Sidney-Filho, L. A., Moreira, A. L. S., de Tarso Dalcin, P., .. W Watte, G. (2018). Predictors of noncompliance to pulmonary tuberculosis treatment: An insight from South America. PLoS One, 13(9), e0202593. https://doi.org/10.1371/journal.pone.0202593

Farha, R. A., Suyagh, M., Alsakran, L., Alsous, M., \& Alefishat, E. (2016). Parental views of antibiotic use in children with upper respiratory tract infections in Jordan. Tropical Journal of Pharmaceutical Research, 15(9), 2009-2016. https://doi.org/10.4314/tjpr.v15i9.27

Foley, M., Carney, T., Rich, E., Dada, S., Mburu, C., \& Parry, C. (2018). A study of medical professionals' perspectives on medicines containing codeine in South Africa. The South African journal of psychiatry, 24, 1162. https://doi.org/10.4102/sajpsychiatry.v24.i0.1162

Gu, J., Zhao, J., Huang, Y., Yang, W., Ren, Z., Li, W., . . Fu, Y. (2015). Use of antibiotics by urban and rural residents in Heilongjiang Province, China: cross-sectional study. Tropical Medicine \& International Health, 20(12), 1815-1822. https://doi.org/10.1111/tmi.12602

Gumede-Moyo, S., Filteau, S., Munthali, T., Todd, J., \& Musonda, P. (2017). Implementation effectiveness of revised (post-2010) World Health Organization guidelines on prevention of mother-to-child transmission of HIV using routinely collected data in sub-Saharan Africa: a systematic literature review. Medicine, 96(40), e8055. https://doi.org/10.1097/MD.0000000000008055

Guo, L., Xu, Y., Deng, J., Gao, X., Huang, G., Huang, J., . . Lu, C. (2017). Associations between childhood maltreatment and non-medical use of prescription drugs among Chinese adolescents. Addiction, 112(9), https://doi.org/10.1111/add.13850

Haddad, M., \& Ebada, M. E. (2017). Demographic and socioeconomic characteristics of outpatients could modify their attitude towards misusing medications in northern Jordan. Journal of Public Health Research, 6(1), 24-30. https://doi.org/10.4081/jphr.2017.818

Inciardi, J. A., Surratt, H. L., Kurtz, S. P., \& Burke, J. J. (2006). The diversion of prescription drugs by health care workers in Cincinnati, Ohio. Subst Use Misuse, 41(2), 255-264. https://doi.org/10.1080/10826080500391829

Jaber, D., Bulatova, N., Suyagh, M., Yousef, A.-M., \& Wazaify, M. (2015). Knowledge, Attitude and Opinion of 
Drug Misuse and Abuse by Pharmacy Students: A Cross-Sectional Study in Jordan. Tropical Journal of Pharmaceutical Research, 14(8), 1501-1508. https://doi.org/10.4314/tjpr.v14i8.25

Lei, Y., Xi, C., Li, P., Luo, M., Wang, W., Pan, S., . . Lu, C. (2018). Association between childhood maltreatment and non-medical prescription opioid use among Chinese senior high school students: The moderating role of gender. J Affect Disord, 235, 421-427. https://doi.org/10.1016/j.jad.2018.04.070

Li, R., Xiao, F., Zheng, X., Yang, H., Wang, L., Yin, D., . . Chen, B. (2016). Antibiotic misuse among children with diarrhea in China: results from a national survey. PeerJ, 4, e2668. https://doi.org/10.7717/peerj.2668

Martinez, P., Zemore, S. E., Pinedo, M., Borges, G., Orozco, R., \& Cherpitel, C. (2019). Understanding differences in prescription drug misuse between two Texas border communities. Ethn Health, 1-17. https://doi.org/10.1080/13557858.2019.1620175

Merz, F. (2018). United Nations Office on Drugs and Crime: World Drug Report 2017. 2017. SIRIUS-Zeitschrift für Strategische Analysen, 2(1), 85-86. https://doi.org/10.1515/sirius-2018-0016

Mun Kit, L., Pauline Siew Mei, L., Sri Ponnampalavanar, S. S. L., Syed Omar, S. F., Taib, N. A., Yusof, M. Y., . . Adeeba, K. (2015). Antibiotics in surgical wards: use or misuse? A newly industrialized country's perspective. Journal of Infection in Developing Countries, 9(11), 1264-1271. https://doi.org/10.3855/jidc.6731

Parry, C. D. H., Rich, E., Van Hout, M. C., \& Deluca, P. (2017). Codeine misuse and dependence in South Africa: Perspectives of addiction treatment providers. South African medical journal, 107(5), 451-456. https://doi.org/10.7196/SAMJ.2017.v107i5.12242

Peng, D., Wang, X., Xu, Y., Sun, C., \& Zhou, X. (2018). Antibiotic misuse among university students in developed and less developed regions of China: a cross-sectional survey. Global Health Action, 11(1), 1-1. https://doi.org/10.1080/16549716.2018.1496973

Pires, D., de Kraker, M. E. A., Tartari, E., Abbas, M., \& Pittet, D. (2017). 'Fight antibiotic resistance-It's in your hands': call from the World Health Organization for 5th may 2017. Clinical Infectious Diseases, 64(12), 1780-1783. https://doi.org/10.1093/cid/cix226

Shirzadeh, E., Shomoossi, N., \& Abdolahzadeh, H. (2016). Topical Anesthetic Misuse in Patients Admitted to Sabzevar Eye Clinic in Iran. Acta Facultatis Medicae Naissensis, 33(4), 287-294. https://doi.org/10.1515/afmnai-2016-0030

Singh, A. R. (2010). Modern Medicine: Towards Prevention, Cure, Well-being and Longevity. Mens sana monographs, 8(1), 17-29. https://doi.org/10.4103/0973-1229.58817

Van Hout, M. C., Norman, I., Rich, E., \& Bergin, M. (2017). Experiences of Codeine Use, Misuse and Dependence: Application of Liese and Franz's Cognitive Developmental Model of Substance Abuse. Behavioural and cognitive psychotherapy, 45(3), 238-252. https://doi.org/10.1017/S1352465817000030

Van Hout, M. C., Rich, E., Dada, S., \& Bergin, M. (2017). "Codeine Is My Helper": Misuse of and Dependence on Codeine-Containing Medicines in South Africa. Qualitative health research, 27(3), 341-350. https://doi.org/10.1177/1049732315613764

Wang, X., Peng, D., Wang, W., Xu, Y., Zhou, X., \& Hesketh, T. (2017). Massive misuse of antibiotics by university students in all regions of China: implications for national policy. International journal of antimicrobial agents, 50(3), 441-446. https://doi.org/10.1016/j.ijantimicag.2017.04.009

Wang, X. M., Zhou, X. D., \& Hesketh, T. (2016). Massive misuse of antibiotics by university students in China: a cross-sectional survey. Lancet, 388(Supp 1 1), S94. https://doi.org/10.1016/S0140-6736(16)32021-9

Wati Ngadimon, I., Islahudin, F., Hatah, E., Mohamed Shah, N., \& Makmor-Bakry, M. (2015). Antibiotic and shared decision-making preferences among adolescents in Malaysia. Patient Preference \& Adherence, 9, 665-673. https://doi.org/10.2147/PPA.S82844

Wells, J. S., Bergin, M., Van Hout, M. C., McGuinness, P., De Pleissisc, J., Rich, E., . . Gooney, M. A. (2018). Purchasing Over The Counter (OTC) Medicinal Products Containing Codeine - Easy Access, Advertising, Misuse and Perceptions of Medicinal Risk. $J$ Pharm Pharm Sci, 21(1), 30049. https://doi.org/10.18433/jpps30049

Yaylaci, S., Tosun, O., Sahin, O., Genc, A. B., Aydin, E., Demiral, G., . . . Varim, C. (2017). Misuse of levothyroxine and the rate of achieving target thyroid-stimulating hormone in levothyroxine treatment. Biomedical Research (0970-938X), 28(6), 2661-2665. Retrieved from 
http://search.ebscohost.com/login.aspx?direct=true \&db=a9h\&AN=122441844\&site=ehost-live

Yong-Kang, C. (2018). Determining Factors of Medication Label Awareness: The Case of Malaysia. Institutions \& Economies, 10(3), 57-80. Retrieved from http://search.ebscohost.com/login.aspx?direct=true\&db=sxi\&AN= 130941768\&site $=$ ehost-live

\section{Copyrights}

Copyright for this article is retained by the author(s), with first publication rights granted to the journal.

This is an open-access article distributed under the terms and conditions of the Creative Commons Attribution license (http://creativecommons.org/licenses/by/4.0/). 